\title{
Eigenfrequencies of vortex state excitations in magnetic submicron-size disks
}

K. Yu. Guslienko ${ }^{1,}{ }^{*}$, B. A. Ivanov ${ }^{2}$, V. Novosad ${ }^{3,4}$, Y. Otani ${ }^{3,5}$, H. Shima ${ }^{3}$, and K. Fukamichi ${ }^{3}$

${ }^{1}$ School of Physics, Korea Institute for Advanced Study, Seoul 130-012, South Korea

${ }^{2}$ Institute of Magnetism, National Academy of Sciences of Ukraine, Kiev 03142, Ukraine

${ }^{3}$ Department of Materials Science, Graduate School of Engineering, Tohoku University, Sendai 980-8579, Japan

${ }^{4}$ Materials Science Division, Argonne National Laboratory, Argonne, IL 60439, USA

5 JST, Japan Science \& Technology Corporation, CREST, Kawaguchi 332-0012, Japan

\begin{abstract}
We have studied theoretically and numerically dynamic properties of the vortex magnetic state in soft submicron ferromagnetic dots with variable thickness and diameter. To describe the vortex translation mode eigenfrequencies we applied the equation of motion for the vortex collective coordinates. We calculated the vortex restoring force with explicit account of magnetostatic interaction on the bases of the "rigid" vortex and two-vortices "side charges free" models. The latter model well explains the results of our micromagnetic numerical calculations. The translation mode eigenfrequency is inversely proportional to the vortex static initial susceptibility and lies in $\mathrm{GHz}$ range for submicron in-plane dot sizes.
\end{abstract}


Introduction. Magnetic solitons and other non-linear magnetization distributions attract much attention in physics of magnetic materials. The magnetization distribution for small ferromagnetic particles (magnetic dots) can be essentially non-uniform. In particular, it is well known that a vortex (soliton-like) state appears for large enough in-plane dot sizes if the dots are made of soft magnetic material. ${ }^{1}$ The vortex magnetization distribution leads to considerable modification of the normal magnon spectrum in comparison with uniform case, in particular, to appearance of low-frequency modes corresponding to displacement of the vortex as a whole. ${ }^{2,3}$ The frequency of this mode was calculated within the exchange model for finite spin lattice in Ref. 3. For proper consideration of the vortex state and vortex dynamic excitations in magnetic dots, however the magnetostatic interaction cannot be ignored because it determines often their physical properties.

In this paper theoretical and numerical investigations of the vortex oscillation mode are presented for magnetically soft (permalloy) disks. We consider sub-micron cylindrical dots with radius $R$ and thickness $L$. The dot thickness is assumed to be about the exchange length of materials, which allows us to neglect the dependence on coordinate along the dot thickness and consider $2 D$ magnetization distribution. The dot magnetostatic energy is accounted explicitly and determines the vortex translation eigenfrequency for submicron-sized dots.

Theory of vortex oscillations. The theoretical description of the vortex oscillations was done on the ground of the effective equation for vortex coordinates. ${ }^{4}$ We consider here only the vortex translation mode. This mode has the lowest frequency in the vortex excitation spectra for small enough dot thickness $L$. We assume two-dimensional magnetization distribution $\mathbf{m}(\tilde{\mathbf{n}}, t)=\mathbf{M}(\tilde{\mathbf{n}}, t) / M_{s}, \mathbf{m}^{2}=1$ within the dot, which does not depend on $z$-coordinate along the dot thickness. We use the angular parameterization for the dot magnetization components $m_{z}=\cos \Theta$, 
$m_{x}+i m_{y}=\sin \Theta \exp (i \Phi), \Phi=q \varphi+\Phi_{0}$. Here $\Phi_{0}= \pm \pi / 2$ corresponds to counter-clockwise and clockwise rotation of vector $\mathbf{m}$ in the dot plane, respectively. The spin structure of static vortex located in the dot center is described by ansatz $\tan (\Theta(\rho) / 2)=\rho / b$, if $\rho<b$ and $\Theta(\rho)=\pi / 2$ if $b \leq \rho \leq R$ suggested in Ref. 5. Here $b$ is the vortex core radius. In order to describe the translation mode of the vortex motion we use collective-variable approach and apply the Thiele's equation ${ }^{4}$

$$
\mathbf{G} \times \frac{d \mathbf{X}}{d t}-\frac{\partial W(\mathbf{X})}{\partial \mathbf{X}}=0
$$

where $\mathbf{X}=(X, Y)$ is the vortex center position, $W(\mathbf{X})$ is the potential energy of the shifted vortex. The first term is the gyroforce determined by the vortex non-uniform magnetization distribution (topological charge). ${ }^{2,3}$ The gyroforce is proportional to the gyrovector $\mathbf{G}=-G \hat{\mathbf{z}}$, where the gyroconstant is $G=2 \pi q p L M_{\AA} \gamma \gamma, \gamma$ is the gyromagnetic ratio, $M_{s}$ is the saturation magnetization. The parameter $q= \pm 1, \pm 2, \ldots$ is vorticity, it determines the direction of the in-plane magnetization components $(|q|$ is the vortex topological charge). $p= \pm 1$ is the vortex polarization (direction of the $m_{\mathrm{z}}$ component in the vortex center). We choose $p=1$ and $q=1$ ( $q=-1$ corresponds to anti-vortex solution), that describes the simplest vortices observed experimentally in remanent state of soft magnetic cylinders. ${ }^{1,6,7}$ The non-zero gyrovector being an intrinsic property of the vortex ${ }^{3}$ is principally important for vortex dynamics description. The gyrovector can be calculated by integration over the vortex core as follows ${ }^{4}$

$$
\mathbf{G}=\frac{M_{s}}{\gamma} L \int d^{2} \tilde{\mathbf{n}} \sin \Theta(\nabla \Phi \times \nabla \Theta)
$$

The second term in Eq. (1) describes the restoring force acting on the vortex shifted from the dot 
Zeeman and anisotropy contributions to the total vortex magnetic energy $W(\mathbf{x})$ in the dot. The restoring force appears due to finite dot in-plane size and is directed toward the dot center. For the submicron dot, radii the dot magnetostatic energy (shifted vortex induces magnetic charges) gives main contribution to $W(\mathbf{X})$-dependence of the vortex centered in equilibrium at $\mathbf{X}=0$.

The vortex static energy dependence on the vortex center position was recently calculated ${ }^{6,7}$ on the basis of the "rigid" vortex model, describing the vortex static susceptibility. The similar calculation was carried out on the "two-vortices" model with no magnetic side surface charges ${ }^{8}$, where the complex function $w\left(\xi, \xi^{-}\right)=\tan (\Theta(x, y) / 2) \exp (i \Phi(x, y))$, with complex variable $\zeta=(x+i y) / R$ was used. The function $w(\xi, \bar{\zeta})$ has the form ${ }^{9} w(\xi, \bar{\zeta})=f(\xi)$ if $\mid f(\xi)<1$ (within the vortex core) and $w(\zeta, \bar{\zeta})=f(\xi) / \mid f(\zeta)$ if $\mid f(\zeta) \geq 1$, where $f(\xi)$ is an appropriate analytical function. In our case $f(\zeta)=(i / c)(\zeta-s)$ corresponds to "rigid" vortex model, $|s|=|\mathbf{X}| / R<<1$ is relative vortex center shift, and $f(\zeta)=(1 / c)\left(\xi \xi+a\left(1-\zeta^{2}\right)\right)$ corresponds to "two-vortices side surface charges free" model ${ }^{8}$ with $(\mathbf{m} \cdot \mathbf{n})_{S}=0, c=b / R$ is the relative core radius, $s=i a$ is the relative vortex center displacement at $a<<1, a$ is real. The coordinates of the vortex centers are connected by the inversion transformation. The parameters $c, s$, and $a$ are determined from the total dot magnetic energy minimization. The values $s=a=0$ correspond to the centered vortex. ${ }^{5}$

For small displacement of the vortex center from its equilibrium position $(\mathbf{X}=0)$ one can write $W(\mathbf{X})=W(0)+1 / 2 \kappa X^{2}$, where stiffness coefficient $\kappa$ can be determined following Ref. 6 from the decomposition of the dimensionless vortex energy $w=W / M_{s}^{2} V$

$$
w(\mathbf{i})=w(0)+\frac{1}{2 \chi(0)} \grave{\mathbf{i}}^{2}-\mathbf{i} \cdot \mathbf{h}+O\left(u^{4}\right)
$$


where $\mathbf{i}=\langle\mathbf{m}\rangle_{S}$ is the dot in-plane averaged magnetization with its projection on the field direction $\mathbf{i} \cdot \mathbf{h} / h=\xi s$, where $\mathbf{h}=\mathbf{H} / M_{\mathrm{s}}$ is the external in-plane field, $V=\pi R^{2} L$ is the dot volume, and the parameter $\xi(\sim 1)$ describes different models of the vortex magnetization distribution.

The vortex initial susceptibility $\chi(0)$ depends on the disk geometrical parameters such as radius $R$ and thickness $L$. For the "rigid" vortex model ${ }^{6} \xi=1$ and $\chi(0)^{-1}=\left(4 \pi F(L / R)-\left(R_{0} / R\right)^{2}\right)$, where $F(x)=\int d t t^{-1} f(x t) J_{1}^{2}(t)$ corresponds to the averaged in-plane dot demagnetizing factor, $f(x)=1-(1-\exp (-x)) / x, J_{1}(x)$ is the Bessel's function, $R_{0}$ is the exchange length. For the "twovortices" model $\xi=2 / 3$ and the susceptibility is $\chi(0)^{-1}=9\left(4 \pi F_{v}(L / R)-\left(R_{0} / R\right)^{2} / 2\right)$, with $F_{v}(x)=\int d t t^{-1} f(x t)\left[\int_{0}^{1} d \rho \rho J_{1}(\rho t)\right]^{2}$.

For the vortex in-plane circular motion the vortex velocity $\mathbf{V}=d \mathbf{X} / d t$ can be represented via the angular frequency vector $\grave{\mathbf{u}}=\omega \hat{\mathbf{z}}$ as $\mathbf{V}=\grave{\mathbf{u}} \times \mathbf{X}$ and thus $\mathbf{G} \times \mathbf{V}=G \omega \mathbf{X}$. The latter equation by accounting $\partial W(\mathbf{X}) / \partial \mathbf{X}=\kappa \mathbf{X}$ leads to the vortex oscillation eigenfrequency $\omega_{0}=\kappa / G$. This frequency for cylindrical dot according to Eq. (3) has the form

$$
\omega_{0}=\frac{1}{2} \gamma M_{s} \frac{\xi^{2}}{\chi(0)}
$$

The eigenfrequency of the vortex oscillations is inversely proportional to the susceptibility and gyroconstant $G$. For typical dot sizes with $\chi(0) \sim 1$ and $M_{\mathrm{s}}=800 \mathrm{G}$ (FeNi dots), the eigenfrequency $\omega_{0} /(2 \pi)$ lies in the $\mathrm{GHz}$ range reflecting the size dependence of the dot initial susceptibility $\chi(0)$. This frequency, however, goes to zero near the critical line of the vortex stability $\chi(0)^{-1}=0$. 
Micromagnetic simulations and discussion. The dynamic behavior of a single magnetic vortex trapped in the circular ferromagnetic dot was separately examined using $2 D$ Landau-LifshitzGilbert (LLG) micromagnetic solver developed by Donahue and Porter. ${ }^{10}$ The computational material parameters are typical for permalloy such as the saturation magnetization $M_{\mathrm{s}}=8.0 \times 10^{5}$ $\mathrm{A} / \mathrm{m}$, and the exchange stiffness constant $A=1.3 \times 10^{-11} \mathrm{~J} / \mathrm{m}$. The unit cell element size is $4 \mathrm{~nm} \times 4$ $\mathrm{nm}$. The dot diameter and thickness are varied from 0.2 to $0.5 \mu \mathrm{m}$, and from 10 to $50 \mathrm{~nm}$, respectively.

Figure 1 shows a time evolution of the in-plane magnetization components obtained with micromagnetic calculation for circular ferromagnetic dot, $0.2 \mu \mathrm{m}$ in diameter and $20 \mathrm{~nm}$ in thickness. First, a magnetic field of $10 \mathrm{mT}$ is applied to the remanent vortex spin distribution, leading to the vortex core precession. The averaged magnetization component $\mu_{\mathrm{x}}$, parallel to the field, oscillates around its equilibrium value of 0.1 , whereas $\mu_{\mathrm{y}}$ oscillates around zero. This precessional behavior can be described in terms of free damped oscillations of vortex core given by Eq. (1). The amplitude of the oscillations with damping decreases with time, and finally the vortex core is stabilized into the new stable position in accordance with the balance among magnetostatic, Zeeman and exchange energies. After removing magnetic field, the vortex core oscillates back to its remanent state.

Figure 2 compares the trajectories of vortex core precession around the remanent state $(\mathbf{X}=0)$ for different damping factors $\alpha=0.2$ (left) and 0.05 (right). With decrease the micromagnetic damping parameter $\alpha$, the total time of oscillations increase, whereas the period (or frequency) remains unchanged. At the limit case of $\alpha=0$, the center of the vortex will oscillate along circular trajectory with the eigenfrequency given by Eq. (4). Note, that direction of the vortex core rotation (counter-clockwise or clockwise) is defined by the combination of the signs of $\Phi_{0}= \pm \pi / 2$, and polarization $p$ as it can be clearly seen in Figs. 1 and 2. The corresponding frequency $\omega$ is positive for $p=1$ and negative for $p=-1\left(\Phi_{0}=\pi / 2\right)$ keeping its absolute value unchanged. 
Figure 3 summarizes the frequencies $\omega_{0}$ of the vortex translation mode determined using the micromagnetic calculations (open symbols) and analytical models (solid lines) given by Eq. (4) as a function of the dot aspect ratio $\beta=L / R$. The analytical results based on the "two-vortices" model with $\xi=2 / 3$ agree well with the micro-magnetic LLG calculations for all the values of $\beta$. The "rigid" vortex model was successfully applied to explain the experimental data of the static initial susceptibility $\chi(0)$ in circular dot arrays, ${ }^{6}$ but it fails to describe the dynamic behavior of the vortex magnetization state. For the submicron dots the eigenfrequency of translation mode depends mainly on the dot aspect ratio $\beta=L / R$. We have approximately $\chi^{-1}(0)=2 \beta(\ln (8 / \beta)-1 / 2)$ for the "rigid" vortex model and $\chi^{-1}(0)=9.98 \beta$ for the "two-vortices" model at $\beta<<1$. Therefore, the vortex translation eigenfrequency is approximately proportional to $\beta$. The susceptibilities are almost equivalent for both the vortex models, thus the translation mode eigenfrequency is mainly determined by value of $\xi$. Strictly speaking, there is total topological charge $q=2$ for the "twovortices" model but in linear approximation on the vortex shift and $c<<1$ we have $q_{\mathrm{ef}}=1$ in Eq. (1). This means that the second, image vortex located outside the dot contributes to the dot magnetic energy $W(\mathbf{x})$, but does not contribute to the gyroforce $\mathbf{G} \times \mathbf{V}$. For large enough $L$, the situation for the vortex eigenfrequencies is quite different: the lowest frequency should correspond to radial oscillation of the dot magnetization (oscillations of the vortex core radius $c$ ) and can not be discussed in terms of the vortex core position.

Conclusions. We have studied dynamic properties of the vortex magnetic state in soft submicron ferromagnetic dots with variable thickness and diameter. The "rigid" vortex and "twovortices side charges free" models were used to define the eigenfrequency of translation mode of the vortex state excitations. The vortex center trajectory for this mode is circular (no damping) or spiral with accounting of damping. The "two-vortices" analytical description is in good qualitative and quantitative agreements with micromagnetic calculations. The eigenfrequency of the vortex tranclation mode ic determined for cuh-micron dots hv the dot acnect ratin $R$ and increaces linearly 
with increasing $\beta$. The angle $\Phi_{0}$ and vortex polarization $p$ influence on the direction of the vortex oscillations only.

Acknowledgments. This work was supported in part by Korea Institute for Advanced Study, RFTF of Japan Society for the Promotion of Science, and the Grant-in-Aid for Scientific Research from the Ministry of Education, Science, and Culture in Japan, and the grant INTAS 97-31311. Work at ANL was supported by US Department of Energy, BES Materials Sciences under contract W-31-109-ENG-38. 


\section{References}

${ }^{1}$ J. Raabe, R. Pulwey, S. Sattler, T. Schweinbock, J. Zweck, and D. Weiss, J. Appl. Phys. 88, 4437 (2000).

${ }^{2}$ G. M. Wysin, Phys Rev. B54, 15156 (1996).

${ }^{3}$ B. A. Ivanov, H. J. Schnitzer, F. G. Mertens and G. M. Wysin, Phys. Rev. B58, 8464 (1998).

${ }^{4}$ A. A. Thiele, Phys. Rev. Lett. 30, 230 (1973); D.L. Huber, Phys. Rev. B26, 3758 (1982).

${ }^{5}$ N. A. Usov and S. E. Peschany, J. Magn. Magn. Mater. 118, L290 (1993).

${ }^{6}$ K. Yu. Guslienko, V. Novosad, Y. Otani, and K. Fukamichi, Appl. Phys. Lett. 78, 3848 (2001).

${ }^{7}$ V. Novosad, K. Yu. Guslienko, Y. Otani, H. Shima, K. Fukamichi, N. Kikuchi, O. Kitakami, and Y. Shimada, IEEE Trans. Magn., EMG-37, 2088 (2001).

${ }^{8}$ K. L. Metlov and K. Yu. Guslienko, J. Magn. Magn. Mat. (2001), accepted.

${ }^{9}$ K. Yu. Guslienko and K.L. Metlov, Phys. Rev. B63, 100403-1 (2001).

${ }^{10}$ M. J. Donahue and D. G. Porter, “OOMMF User's Guide, Version 1.0”, Interagency Report NIST IR 6376, National Institute of Standards and Technology, Gaithersburg, MD (1999). 


\section{Figure captions}

\section{Fig. 1}

Time-evolution of $\mu_{\mathrm{x}}$ and $\mu_{\mathrm{y}}$ magnetization components in applied in-plane field of $10 \mathrm{mT}$ and in remanence. Solid and dashed lines correspond to the vortex magnetic state with polarization $p=1$ and -1 , respectively. The vorticity $q=1$, the dot radius $R=0.1 \mu \mathrm{m}$, and the thickness $L=20 \mathrm{~nm}$.

\section{Fig. 2}

The trajectories of vortex core precession around the remanent equilibrium position for different damping factors $\alpha=0.1$ (left) and 0.05 (right). The dot geometry is the same as in Fig. 1 .

Fig. 3.

Micromagnetic (markers) and analytical (solid lines) calculations by Eq. (4) of the eigenfrequencies of vortex translation mode $v s$. the dot aspect-ratio $\beta=L / R$. a) the "rigid" vortex model, $b$ ) the "two-vortices" model. 

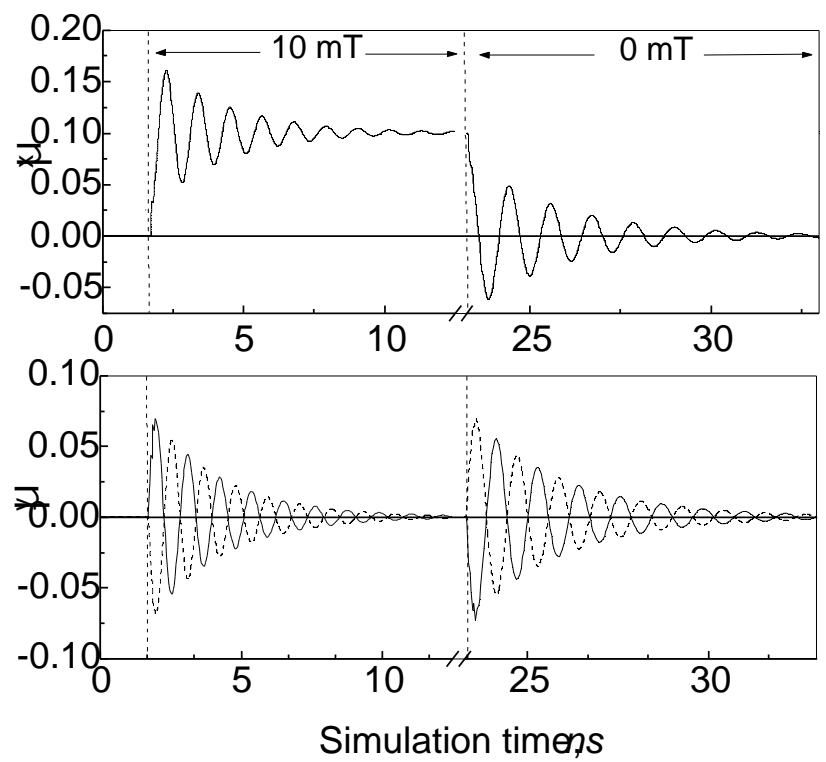

Fig. 1 


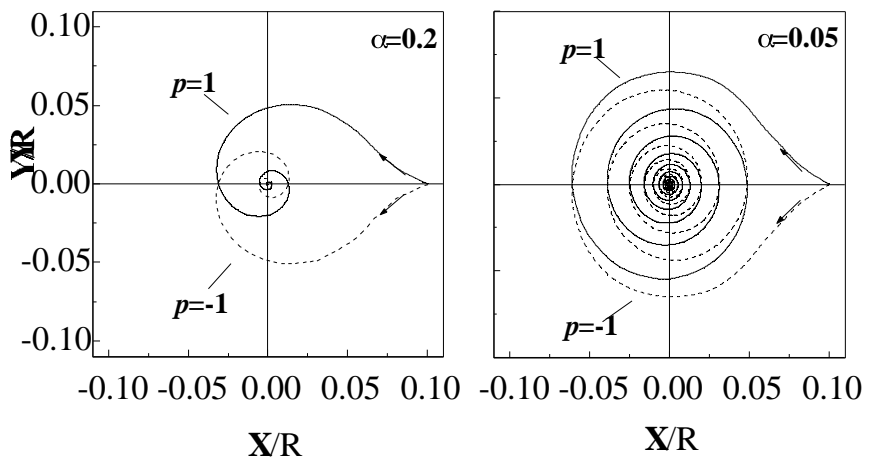

Fig. 2 


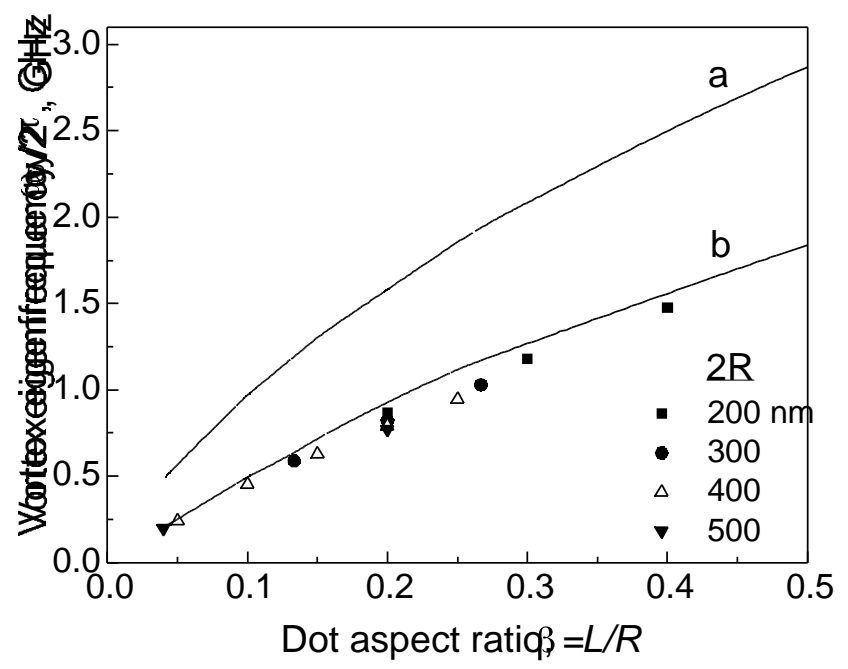

Fig. 3 